thinning by ulceration or by long-continued distension; otherwise the accident would be much more common. Such cases show conclusively to my mind that it is folly to permit patients with distended gall-bladders, even though symptoms be only occasionally present, to go unoperated on. I know of several such cases where patients are living in a "fool's paradise" owing to such unsound advice. A careful operation in these cases is almost devoid of risk, but rupture is hazardous in the extreme. Massage in cases of distended gall-bladder I look on as the height of folly, though it has been advised by those who should know better. Attempts to force impacted calculi onward by pressure are well calculated to rupture the thinned wall of the gall-bladder or bile-ducts or to cause perforation through the base of an ulcer, leading to extravasation of germ-containing fluid into the general peritoneal cavity and probably to fatal peritonitis. In some cases the primary perforation may lead to the formation of a second cavity bounded by plastic lymph, which may again rupture and lead to a fatal peritonitis. The perforation of the gall-bladder may occur into adjoining parenchymatous organs, and on several occasions I have removed numbers of gall-stones from cavities in the liver produced by ulceration and perforation of the gall-bladder or bile-ducts and direct passage of the contents into the liver tissue. In such cases there are the usual signs of liver abscess following on the ordinary symptoms of gall-stones, which may have been present for years. If the ulceration and perforation occur from the common duct into the substance of the pancreas, acute pancreatitis may follow. If the ulceration advances towards the adjoining hollow viscerastomach, duodenum, or colon-adhesions as a rule form and the perforation is effected quietly. In one case of this kind which I saw with Dr. Stewart, after a history of cholelithiasis followed by severe stomach symptoms, the gallstones were vomited 'and complete recovery followed. In several cases I have seen large gall-stones ulcerate their way quietly into the intestine, only producing serious symptoms from mechanical intestinal obstruction. Rarely gall-stones have perforated into the pelvis of the right kidney, producing symptoms of renal calculus. Not infrequently the perforation may occur after adhesion to the parietal peritoneum, when the events described under simple empyema of a superficial abscess discharging gall-stones may follow. The symptoms of perforation of the bile passages are those of perforative peritonitis from other causes, but there will usually have been premonitory symptoms pointing to the origin of the disease. A sudden pain beneath the right ribs, of ten followed by collapse and usually succeeded by vomiting, general distension of the abdomen, and a rapid pulse form the prominent features of the disease. If the extravasation is extensive there will be signs of free fluid in the peritoneal cavity. Jaundice, if not present before the accident, usually comes on from absorption of biliary pigment by the peritoneum, and if the bowels can be moved the motions will usually be clay-coloured. If the case be not operated on, death will probably ensue within a few days from toxæmia and paralysis of the bowels, though in some of the cases I have quoted life was prolonged into the second or third week. As to treatment, in these cases medical treatment is useless, and giving opium for the relief of pain so disguises the symptoms that a fatal sense of security is given for a time, and when the mistake is discovered it is too late to operate. As soon as it is clearly maje out that perforation has occurred, or even if it be suspected that such is the case, the abdomen should be opened in the right semilunar line. If pus and bile be found they should be rapidly wiped away with gauze or wool sponges, and if the extravasation has gone beyond the local area of disease the abdomen should be flushed with hot boracic lotion. The patient may be too ill to bear a prolonged operation, and if so free drainage, as in the case I have related, will probably do all that is necessary. In draining it may be borne in mind that the right kidney pouch forms a distinct peritoneal pocket, and that a drainage tube applied through a stab opening in the right loin affords a free exit for extravasated fluids coming from the neighbourhood of the gall-bladder. If the whole peritoneal cavity has been soiled a puncture above the pubes large enough for a tube to be passed into Douglas's pouch may be an advantage. If the patient be in sufficiently good condition to permit a search for the rupture, and it can be found, it may be closed by fine silk or catgut sutures, but as a rule it will be wise to open and drain the gall-bladder at the same time. Should marked cholecystitis be found the question of cholecystectomy may be worth considering, but when the patient is in a critical condition it is a mistake to attempt too much ; and as a rule cleansing and free drainage will be all that are necessary or advisable at the time, the removal of the cause being left until the patient is better able to bear a prolonged operation.

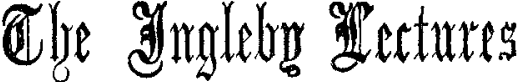

ov

\section{THE OPERATIVE TREATMENT OF CANCER OF THE BREAST.}

\author{
Detivered at Mason College, Birminglam, on May 19th \\ and 26th.
}

By BENNETT MAY, F.R.C.S. ENG., JOINT PROFESSOR OF SURGERY IN MASON COLLEGE, BIRMINGHAM.

\section{LECTURE II. ${ }^{1}$}

Delivered on May $26 t h$.

GENTLXMEN, - I shall now proceed to consider the results which have been obtained from this method of operating, adopting Volkmann's three years limit as the standard of a cure for comparison with former methods. This means that the patient shall have had a full period of three years or longer after operation without local or internal recurrrence. Experience shows that the prospect of permanent immunity is then considerable. At the same time $I$ do not accept this-or, indeed, any other period-as at all absolute, owing to the way in which minute deposits may temporarily remain quiescent and latent. I have seen external recurrence after an interval of eleven years, and not infrequently a patient dies from the disease five, six, or more years after operation, though in these cases I think some sign might have been detected within the three years limit. However, in this limited and provisional sense, I accept it as a cure. Then a distinction is to be drawn between local external recurrence and internal metastatic deposit as evidence of completeness of operation. The efficiency of the operation is more correctly measured by freedom from local recurrence than internal deposit, as the former is evidence that the operative limits were reached and nothing further could have been done. Moreover, it is supposed that cases are taken without special selection so long as they are operable. I believe the following is an exposition of the best work which has been done in the treatment of cancer of the breast on the lines of the radical operation described. The following table shows the recently published returns of three operators :-

TABLE A.-Cases operated on by the Radical Method and observed for at least three years.

\begin{tabular}{|c|c|c|c|c|c|c|}
\hline \multirow{2}{*}{ Surgeon. } & \multirow{2}{*}{$\begin{array}{l}\text { No. of } \\
\text { cases. }\end{array}$} & \multicolumn{2}{|c|}{$\begin{array}{l}\text { No local } \\
\text { recurrence. }\end{array}$} & \multirow{2}{*}{$\begin{array}{l}\text { Local } \\
\text { recur- } \\
\text { rence. }\end{array}$} & \multirow{2}{*}{$\begin{array}{c}\text { Per- } \\
\text { centage } \\
\text { of } \\
\text { cures. }\end{array}$} & \multirow{2}{*}{ 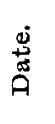 } \\
\hline & & Oured. & Died. & & & \\
\hline $\begin{array}{llll}\text { Rotter } & \ldots & \ldots & \ldots\end{array}$ & 10 & 6 & 2 & 2 & $60 \cdot 0$ & 1896 \\
\hline Helfer ich & 35 & 10 & 11 & 1.4 & $30 \cdot 5$ & 1896 \\
\hline Watson Cheyne ... & 21 & 12 & 4 & 5 & $57 \cdot 0$ & 1896 \\
\hline Iotal ... & 66 & 28 & 17 & 21 & $42 \cdot 1$ & $\rightarrow$ \\
\hline
\end{tabular}

Percentage of cures (average) $42 \cdot 1$; percentage with no local recurrence 68 ; operative mortality 0 .

Von Bergmann does a bold operation on ordinary lines, removing the breast widely with the paramammary tissues, systematically clearing the axilla, and in some cases removes the sternal portion of the pectoralis major. His latest statistics, however, do not come down later than 1885, when he was less careful about clearing the fascia, \&c., than now, and I have not included them in this table. Rotter (Berlin) does a wide operation. He removes the whole of the pectoralis major and the pectoralis minor. He also removes the

1 Lecture I. appeared in The LAxckT, May 22nd, 1897. 
clavicular part of the major when the infra-clavicular glands are enlarged. In some cases he removes the supra-clavicular giands, but does not resect the clavicle. Helferich (Griefswald) does a similar operation, but without removing the pectoralis minor. Watson Cheyne doss a wide, clean operation on ordinary lines, occasionally removing the sternal portion of the pectoralis major. $\mathrm{He}$ does not ordinarily remove the supra-clavicular glands. His results seem to be the best recorded, viz., 21 cases with 12 cures-57 per cent.

TABLE B.-Statistics showing Gradual Improvement in Results.

\begin{tabular}{|c|c|c|}
\hline Operator. & Date. & Percentage of cures. \\
\hline $\begin{array}{llllll}\text { Billroth } & \ldots & \ldots & \ldots & \ldots & \ldots\end{array}$ & 1876 & $4 \cdot 7$ \\
\hline $\begin{array}{llllll}\text { Esmarch } & \ldots & \ldots & \ldots & \ldots & \ldots\end{array}$ & 1876 & $11 \cdot 8$ \\
\hline $\begin{array}{lllll}\text { Volkmann } & \ldots & \ldots & \ldots & \ldots\end{array}$ & 1880 & $17 \cdot 8$ \\
\hline $\begin{array}{llllll}\text { Kuster } & \ldots & \ldots & \ldots & \ldots & \ldots\end{array}$ & 1885 & $21 \cdot 0$ \\
\hline $\begin{array}{llllll}\text { Konig } & \ldots & \ldots & \ldots & \ldots & \ldots\end{array}$ & 1885 & $23 \cdot 0$ \\
\hline $\begin{array}{llll}\text { Von Bergmann } & \ldots & \ldots & \ldots\end{array}$ & 1885 & $32 \cdot 0$ \\
\hline 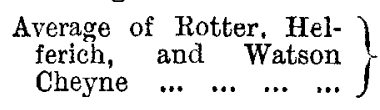 & 1896 & $42 \cdot 5$ \\
\hline
\end{tabular}

Looking to my own results $I$ have seen that the effect of thorough removal is very marked indeed. I find it almost impossible to present these in a complete tabular form principally owing to the difficulty of tracing the hospital patients. I commenced to clear out the axilla as an addition to the ordinary operation in selected cases about 1887. These earlier ones, though not available in statisticial form, were of great value to me, and were largely instrumental in forming my opinion. Thus a woman whose breast and axilla I cleared in May, 1888, for a small scirrhous tumour, microscopically verified, is now alive and perfectly well. Another, operated on in April, 1889, in the same way is perfectly well to-day.

From the commencement of 1891 I have cleared out the axilla in every case of cancer that I have operated on with one exception, viz, a very feeble old lady, aged seventy years, with a small hard scirrhous nodule who presented anæsthetic difficulties. This has been the only exception. Since that date I have performed the complete operation more or less perfectly on seventy-eight patients, forty-four in private practice, and thirty-four in hospital. The ages ranged from twenty-eight to seventyfive years. There was not one death from operation, nor one even seriously ill so as to give anxiety. It is right to say that I have had one death from breast excision for a simple tumour, due to acute septic infiltration in hospital. I have found it impossible to trace the subsequent histories of a sufficient number of the hospital cases dated three years back to make them of any value for statistical purposes so they must be put aside altogether. Of the forty-four private cases I have a report up to date in every one. These cases are divisible into two periods, the first prior to May, 1894, and the second subsequent to that date. Those in the first group only are available for the three years limit, and they are 20 in number. Of these 7 are now alive and free from any sign of the disease, 11 have died from it, and 2 are alive, but show signs. Of the 13 in whom the disease has re-appeared it was solely internal in 7. I should state that this epoch contained an unusual proportion of bad cases, of complete failures and very early recurrences. I have no doubt the 24 in the second epoch will show much better resulta. In the first place I have continuously improved upon my method of operating, particularly in respect of wider removal of muscle and paramammary tissue. Then the selection of cases was more careful and judicious. Thus of 9 operations (private) in 1896 only 1 shows any sign of re-appearance, of 7 in 1895, 1 died within eight months, and 1 shows signs of internal deposit. All the others are quite well.

The adrantages which have followed thorough and systematic operations are, I think, sufficiently apparent, and have fully established their superiority over the older methods. An important dissentient is to be noted in Mr. Frederick Treves, who considers that practically the results are no better than by the older methods while being more tedious and dangerous, but as he allows an average mortality of 5 per cent. for these older ones, and an average re-appearance within twenty months, it is clear that be does not do justice to the possibilities of the newer method while he considerably exaggerates its dangers and drawbacks, its average mortality being below 5 per cent., and immunity from return far more than twenty months.

I think it is more difficult to decide upon the merits of some of the more extensive operations which have recently been introduced, especially by our Transatlantic brethren. Halstead and Meyer do not consider the operation complete unless it includes also the whole of both pectoral muscles, every trace of fatty and connective tissue in the axilla and sub-pectoral region, and the supraclavicular glands in the posterior triangle of the neck. Halstead's operation is rather startling in its extent and magnitude. The technique is not quite easy to understand, but it is fully explained in his article in the "Annals of Surgery," 2 and also described by Jacobson in the last edition of his admirable work on "Operative Surgery." Suffice it to say that Halstead encircles the breast by a very wide incision which is carried below the clavicle on to the arm. A flap of skin is reflected down from the axilla and chest-wall, and is subsequently slid up to form a lining for the fornix of the axilla. Another incision bisecting the main one is carried over the clavicle to the neck and the flaps of skin are reflected. Botk pectoral muscles are detached from the ribs and clavicle and the humerus and the cleaning of the axillary contents is commenced and carried from above downwards. The whole of the tissues are dissected away with scrupulous care, not a trace being left behind, and the whole mass is removed in one piece so that cancerous tissue is never cut into. He now also adds to this a dissection of the supra-clavicular glands from the posterior triangle of the neck. It is described as a bloodless operation, as many as 100 or more ligatures being required, and the time occupied is said to be three or four hours. He claims for it but little shock, as he allows no loss of blood, and as he has done it fifty-one times without a death he is entitled to do this. Halstead distinguishes between local and regionary recurrence. By the former be means return of the disease in the field of operation, in the scar, or in the glands above the clavicle. By regionary recurrence he means skin metastasis at a distance from the scar, and which he believes appear as independent formation being against the lymph current. The distinction, however, cannot well be maintained and seems superfluous. Of his 51 operations Halstead claims that there has been true local recurrence only in 3 , but 8 have shown regionary recurrence, or 11 in all. In 5 the result was not known. The remainder, 34 in number, showed no external recurrence. Of these 10 had died presumably from internal metastasis, so that 24 or 50 per cent. may be considered as cured. The weak point in these statistics is that they are not based upon the three years or any limit-in fact, it does not appear that the three years' limit has been passed in more than three or four of them, a large number being within a few months of the publication. In any case they do not show better results than by the ordinary complete operation such as Watson Cheyne's. It is clear that in both cases the majority that die do so from internal metastasis without local recurrence. The operation seems to be one which may well be reserved for advanced cases where the muscles are infiltrated and the infra-scapular and cervical glands are enlarged, if, indeed, it is thought advisable to touch such cases at all. I believe it was not Halstead's intention to publish any more results till the three years had passed, so that a further and more valuable record should shortly be due.

Meyer's operation goes even further. He makes the usual oval incisions on a wide scale and bisects the upper by a vertical one carried up over the clavicle to the neck. The integumental flaps are dissected well back. Both the pectoral muscles are then removed entire, commencing at the humeral attachment of the major and throwing both inwards. These with all the infra-clavicular and axillary fat and lymphatics are carefully cleaned away with the knife absolutely and continuously working round the seat of disease, and never trespassing even on the belly of the muscle. The supra-clavicular glands are also dissected out of the posterior triangle of the neck through the vertical incision. Any defect of skin is made up by grafting in eight or ten days. This operation, he claims, adds only twenty minutes to the ordinary one, and the disability afterwards is not great. At the time of publication he appears to have done it only in one case, a few months previously, so that no 
opinion can be formed of its special merits. Further than this it is scarcely possible to go without amputating the entire upper extremity with the shoulder girdle. I believe this has been done a few times, though with indifferent success. It is clearly inadmissable, and can only be condemned as a dangerous and useless mutilation without corresponding advantage. Then at least one operator has removed the sternal glands from within the chest, a procedure which is not likely to be frequently followed. Heidenhain also includes the periosteum in stripping the muscles from their bony attachments.

There is diversity of view as to the treatment of the supraelavicular glands. This is certainly our weakest point, and the most common seat of external recurrence, just as the axilla used to be. My own opinion is that if enlarged glands can be felt above the clavicle there is no hope of cure and the case is not suitable for operation. It is to be noted that these supra-clavicular growths often appear to grow very slowly, and that owing to the roomy space and fat in which they lie a long time may elapse before they show any sign of enlarging. Consequently by the time this has taken place the chain of glands beyond them, which is close to the lymphatic duct and passes into the chest. will be extensively infected, and the disease will have passed into a region where it cannot be followed. How far it should be carried as an addition to the ordinary operation is as yet somewhat doubtful. To clear out the posterior triangle of the neck means a considerable addition to the operation and is frequently unnecessary. If, however, it is found during the operation that the highest axillary glands are infected it is clearly necessary to follow them into the neck, unless the case is to be forthwith abandoned as hopeless. There is then a reasonable prospect of getting beyond the limits of the disease. It is not considered that divison or resection of the clavicle materially helps the exposure and removal.

I think it is very doubtful whether some of these more extensive operative procedures confer any additional immunity from recurrence at all commensurate with their severity. It is clear that in both classes of operation the majority of the fatal cases die from the internal metastasis without external recurrence, and against internal metatasis no operation can protect. It is very certain that but little good can result from these extensive measures if there exists some deep-seated nidus of infection which is beyond the reach of the knife. That such a source of infection exists is in many cases only too certain, though not apparent. Herbert Snow finds it in the bone marrow. He claims that this is the great obstacle to radical cure in breast cancer. This infection, he states, takes place in all ordinary cases within six months of the inception of the disease. The typical indication is a prominence of the sternum, though there may not be any more visible evidence for many years, the disease remaining quiet and latent. There is considerable force in his views, though probably much too sweeping. I have on several occasions recognised this enlargement of the sternum as an unfavourable prognostic sign, indicating bone infection.

So far there is no conclusive evidence that any corresponding advantage accrues from some of the more recent developments of the operation, and I am of opinion that the utmost benefit of which it is capable may be conferred in a large majority of cases by the one I have described as the standard. The cases for which this will not suffice are probably unsuited for operation. At the same time, final judgment must be suspended till we receive further reports of these procedures.

The clinical varieties of cancer of the breast vary, as is well known, within wide limits, and appear to me to depend very much on the age, habit of body, and constitution of individual patients. At one extreme is the large, soft, rapidly growing and vascular tumour known as medullary cancer, which occurs in robust, healthy young women, and in stout women of more mature years. These cases are most unfavourable for operation and, except in their earliest stages, are quite unsuitable. They furnish a large proportion of the early recurrences and failures, as the disease sometimes re-appears before the healing of the wound and spreads with great rapidity. Of all the unfavourably modifying conditions I think rapidity of growth is the worst. The actual raration is by no means the only criterion of the stage of e disease. A large tumour of the tuberous forms, of slow 0 vth and unattached, has a much more favourable prognosis than an infiltrating medullary one in its earliest stages. At the other extreme is the small chronic dry infiltration of shrivelled and aged women, though not confined to old women. Opinions differ as to the propriety of operating in atrophic cancer. In my own opinion, it is a question of the stage of the disease. In an early stage they are the most favourable of all and should certainly be removed. Later, when there is any notable skin infiltration or attachment to the muscles, the most radical operation is not only ineffectual, but harmful. It is not only that an early reappearance may be expected, but the type of the disease is thereby changed and the recurrent malady may run its course in as many months as it would have occupied years if undisturbed. I have known a case extend over thirteen years of fairly comfortable life. Atrophic cancer has a remarkable faculty of attaching itself to surrounding parts, and of reproducing fibroid indurations in its recurrences, both regionary and metastatic.

On Paget's discase. - I have had four cases of this peculiar cancerous dermatitis of the nipple. I should adrise immediate operation in every case of chronic eczema or ulcer of the nipple, even though no tumour is perceptible, as the relationship between this and cancer is constant. In all my cases the ulceration preceded the tumour by several years. In two of them the tumour was limited to the nipple, and of the kind known as duct cancer, and preceded by a bloody discharge from the nipple-a symptom of very grave significance.

On secondary operation.-Secondary operations are advisable only when the growths are small, solitary, and freely movable. I think these operable cases generally imply an imperfect primary operation, and should get rarer as we make this more efficient. In two of my cases only have I thought it advisable to remove recurrent nodules by secondary operation, and in neither was the result very satisfactory, as general infiltration soon showed itself. I have seen but one case, and that in the practice of a predecessor, which was greatly benefited by repeated operations, two small nodules being removed from the scar at intervals of four and three years respectively.

On the complete operation.-All cases should be subjected to the complete operation even in their earliest stages. Except as a palliative in a few rare cases and for benign tumours the incomplete operation-that is, of the breast alone-should be abandoned. The additional risk to life is scarcely appreciable, and the advantage of clearing the axilla, the next resting place of the disease, is obvious. After incomplete operations re-appearance takes place in at least 80 per cent. within six months. Even as a palliative the complete operation is much more effective, in particular in preventing that very distressing feature, odema of the upper extremity. It is occasionally necessary to perform a palliative operation on a patient past hope of cure for the sake of the moral effect. To some women the verdict "too late" comes as a crushing blow, and it is more humane to yield to their request if this can be done with any prospect of relief. For instance, we may get rid of a foul and bleeding ulcer while the patient's spirits are maintained by hopefulness.

On the limits of the operation for cure and non-operable cases.-A judicious and careful selection of cases should be exercised and involves consideration of the constitutional state of the patient as well as the local conditions. Among non-operable cases I class: (1) large medullary cancers (2) scattered tubercles in the skin and odema; (3) internal metastasis of all kinds; and (4) odem $a$ and solid enlargement of the upper limb. This I regard as an emphatic "Noli me tangere" and contra-indication. This waxy cedema is one of the most distressing features of the disease I am sorry to say I know no means of relieving it. I have tried puncture, but it is worthless. Amputation at the shoulder girdle has been practised on a few occasions, and I have been asked by patients to do this; but even this heroic remedy does not arrest the progress. Among debatable cases I include:-1. Enlargement of supra-clavicular glands. I have hitherto regarded this as an absolute contra-indication, but I am inclined now to extend the field of operation to some of these cases. I would limit it, however, to small and movable tumours or to the condition I previously indicated of the highest axillary gland being found infected. Fixed and painful enlargements, with odema of the limb, should not be touched. Before long we may expect some further evidence of the merit of this addition to the operation, as it is being extensively practised by some surgeons. 2. Cancer in both breasts. The appearance of the disease in the other breast is now commonly regarded as a late general infection 
and not as an independent growth. It is explained by the free anastomosis and intersection between the lymphatics of the two breasts. It is rarely desirable to operate, but Jacobson records a satisfactory case. The surgeon must consider the probabilities in each case with the interval that has elapsed and the conditions of the re-appearance. It happened in two of my patients. In one of them I operated on the second breast with unsatisfactory results. In the other I did not operate on the second breast; the disease reappeared in this case two years after removal of the first, and, very curiously, it was preceded by enlargement of the axillary glands on the corresponding side.

Diagnosis. - The importance of early operation, obvious as it is, cannot be too strongly enforced. It is unfortunate that the disease is frequently so insidious in its onset that considerable advance has been made before the patient is aware of its existence. Another great hindrance to early operation is the difficulty of diagnosis in the incipient stages. The first care should be to place the patient in favourable circumstances for the examination. The chest should be quite undressed, so as to admit of easy examination of the pectoral region and axilla as well as of both breasts. In a matter of this urgency and importance accuracy of diagnosis should be subsidiary to promptitude in treatment, as there are cases in which it is impossible to be accurate without exploratory incision. The two conditions which give the greatest trouble are chronic inflammatory hyperplasia, and the case of a tense cyst situated deeply in the breast. The latter is distinguished by its mobility, globular outline, and verified by the exploring needle, which should never be overlooked as an aid to diagnosis. Chronic mastitis is often much more difficult to discriminate, and some experience in handling and exploring these indurations is needed. Students are taught to recognise cancer by the well-known evidences in a develoced stage, when the malignant characters are patent to observation; and we are all more or less under the influence of these teachings. In the simple disease which often follows lactation or weaning the thickening is sensitive to the touch, firm, and elastic, bat the outlines are more diffused than in cancer. On pressing the mass against the chest wall the induration and sense of tumour disappear. Then, again, the area of breast occupied is more diffuse and larger, or both breasts may be similarly affected. The same sort of dimpling of the skin may occur as in cancer, but this is quickly followed by fluctuation and suppuration.

It is prudent to come to a decision at the first interviewthat is to say, if the surgeon is unable to convince himself of the non-malignant nature of the neoplasm it is better to take action at once. I deprecate the practice of keeping cases under prolonged observation to see how they will develop. Patients either get restive and will not consent to remain long in doubt, or, what is more common, if told to report themselves in a month they turn up in six months, when the mischief is done. Moreover, the sequel in about five cases out of six shows the delay to have been unwise.

A very important question arises as to the existence of any direct etiological relationship between chronic mastitis and cancer. It is generally assumed that such a relationship exists, that chronic hyperphasia is likely to become cancer, but the stages of development from this supposed precancerous state have not been traced. There seems distinct clinical proof that cancer may be grafted on to it if prolonged. I believe this did take place in the case of one of my patients. This woman, aged forty-eight years, had chronic mastitis, and after an interval of three and a half years from its first incidence a small scirrhous nodule appeared on the site. This made me regret being too precise and nice in my diagnosis. There can be no doubt that before proceeding to do one of these wide operations we must first positively satisfy ourselves that the disease is malignant. In a large proportion-too many, indeed-this is only too obvious. In doubtful cases a preliminary exploratory incision is to be made, and precaution is to be taken against auto-infection. The knife should be changed and the hands of the operator as well as the surface of the breast washed and the incision plugged with sterile gauze. Can we rely upon the naked eye appearance of the cut surface? I think so. The fresh cut section of cancer is unmistakeable, quite different from the leathery toughness of chronic mammitis. Some contend that the surgeon, if not an experienced pathologist himself, should always be accompanied by one with his freezing microtome. This, however, is a luxury which cannot always be had, unless he is to be an official provided at State expense, as suggested in America. One is very glad of the opinion of a skilled pathologist, but in case of a conflict of opinion the responsibility of deciding and acting would remain with the operator.

In closing, let me summarise by saying that so far as is at present known our only hope of advancement lies in the direction of more thorough and more early operation. The limit of what is possible in the former direction will soon be, if it is not already, reached. The result must carry conviction that we may hopefully anticipate a real cure in at least 30 or 40 per cent. or, some would say, 50 per cent. of our cases. I would not like to place 30 per cent. as a limit, but it is with somewhat chastened hope that I look for anything beyond. Longer observation is required, particularly as to the future development of those cases which pass the three years limit. Even now, however, the operation in too many cases is not practised to the best advantage and is not used for all it is worth. Certainly some of the disrepute and prejudice which have surrounded it may fairly be ascribed to the incomplete and inadequate manner in which it is too often done by men who have had no proper surgical training, and whose ill results serve to injure the cause as a whole, and to reflect prejudicially on the work of others. The fact is it has been everyone's operation because it is thought to be easy, but now that surgery is specialised to such an extent these easy happy-go-lucky methods should be abolished in this as in other branches of surgery.

In respect to the second point-more early operation-much has yet to be accomplished. I think, however, that I observe some improvement in this direction and that we now operate at a somewhat earlier average stage than was customary fifteen or twenty years ago. I gladly testify to the alertness of the profession in this respect. When undue delay occurs it is usually the fault of the patient who has concealed it from herself, her friends, and her medical attendant till too late. For this the well known want of success of the operation is partly responsible. If we can show better results women will probably be less reluctant to undergo treatment. I think in lay circles as well as medical there is an increasing recognition of the value of early and complete operation as offering the only hope of cure. For the present all pretensions to influence the course of the disease by other measures seem to have subsided, and one after another the worthless cancer cures have ceased to distract the public mind.

In thanking you for your kind attention I am too well aware that I have added nothing of novelty or weight to what has already been better said by others, but I hope some service may have been done by showing that the operation deserves more careful study and attention than it commonly receives. British surgery has been rather lax in its treatment of breast cancer, and in particular in our own district I think we have much leeway to make up.

\section{TYPHOID SERUM REACTION.}

\section{BY W. C. C. PAKES, M.R C.S. ENG., L.R.C.P. LoND., D.P.H. CANTAB.}

ASSISTANT DEMONSTRATOR OF BACTERIOLOGY AT GUY'S HOSPITAL.

(From the Laboratory at Guy's Hospital.)

ALTHOCGH the typhoid serum reaction has been very generally viewed with favour by most observers as a test for enteric fever, some do not consider it to be of such value. There seems to be a tendency on the part of nearly all to admit that the reaction must be accepted with certain reservations, particularly when a negative result is obtained. It was in order to study the conditions which may be responsible for the different and somewhat anomalous results that I began a series of observations, during the course of which I have examined the sera of sixty different subjects. A brief résumé of the methods and conclusions of some of the different observers will help to explain why I adopted a particular technique.

Grünbaum, ${ }^{1}$ who appears to have been the first to study 